\title{
Spektrum Dermatologie - wissenswert, kompakt, anregend
}

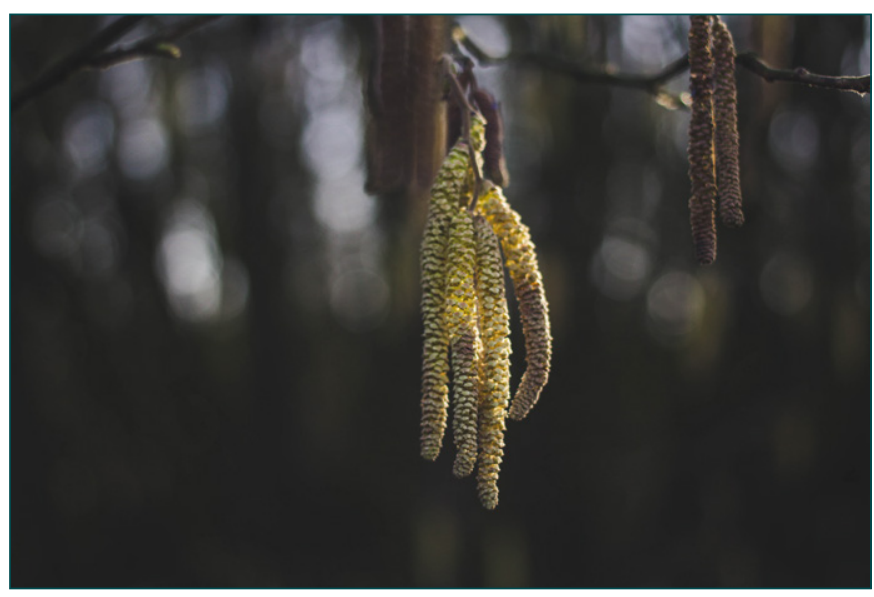

Luftgetragene Pollen erklären im Durchschnitt 44 Prozent der Varianz der Infektionsraten

Foto: Akin auf Unsplash

Technische Universität München

\section{Covid-19-Risiko steigt bei Pollenflug}

Fliegen viele Pollen in der Außenluft, kommt es zu erhöhten Infektionsraten mit SARSCoV-2. Dies hat ein internationales Team unter der Leitung von Forschenden der Technischen Universität München (TUM) und des Helmholtz Zentrums München mit einer breit angelegten Studie gezeigt. Angehörige von Hochrisikogruppen könnten sich durch das Beobachten von Pollenflugvorhersagen und ein entsprechendes Tragen von Staubfiltermasken schützen.

Unter der Federführung von Erstautor Athanasios Damialis sammelte das Team am Lehrstuhl für Umweltmedizin an der TUM Daten zu Pollenkonzentrationen in der Luft, zu meteorologischen Bedingungen und zu SARS-CoV2-Infektionen - dabei wurden die Variationen der Infektionsrate von Tag zu Tag oder auch die Gesamtzahl positiv Getesteter berücksichtigt. In ihre Berechnung bezogen die Wissenschaftlerinnen und Wissenschaftler auch Daten zu Besiedelungsdichte und zu Effekten von Lockdowns ein. Die 154 Forschenden analysierten Pollendaten von 130 Stationen in 31 Ländern auf 5 verschiedenen Kontinenten.
Das Team zeigte, dass luftgetragene Pollen im Durchschnitt 44 Prozent der Varianz der Infektionsraten erklären können - manchmal spielten hier aber auch Luftfeuchtigkeit und Lufttemperatur eine Rolle. An Orten ohne Lockdown-Regelungen stieg die Infektionsrate im Schnitt um 4 Prozent, wenn sich die Anzahl der Pollen in der Luft um 100 pro Kubikmeter erhöhte. Der Grund: Wenn Pollen fliegen, reagiert die Körperabwehr in abgeschwächter Form auf Viren der Atemwege, die verantwortlich für Schnupfen und Erkältungen sind. Wenn ein Virus in den Körper gelangt, produzieren infizierte Zellen üblicherweise Signalproteine - auch bei SARS-CoV-2. Diese sogenannten antiviralen Interferone rufen benachbarte Zellen dazu auf, ihre antivirale Abwehr zu verstärken, um die Eindringlinge in Schach zu halten. Außerdem wird eine ausbalancierte Entzündungsreaktion aktiviert, um die Viren zu bekämpfen.

Ist allerdings die Pollenkonzentration in der Luft hoch und werden neben Viren auch Pollen eingeatmet, werden weniger solcher antiviralen Interferone produziert. Auch die eigentlich heilsame Entzündungsreaktion wird beeinflusst. Wenn viele Pollen fliegen, kann die Zahl der Atemwegserkrankungen daher ansteigen - dies gilt auch für Covid19. Dabei spielt es keine Rolle, ob Betroffene an Allergien gegenüber diesen Pollen leiden oder nicht.

«Man kann nicht vermeiden, luftgetragenen Pollen ausgesetzt zu sein», sagt Stefanie Gilles, ebenfalls Erstautorin der Studie. «Personen, die zu Hochrisikogruppen gehören, sollten deshalb darüber informiert sein, dass erhöhte Pollenkonzentrationen in der Luft anfälliger gegenüber viralen Infekten der Atemwege machen.»

Was also können Personen, die Risikogruppen angehören, tun, um sich zu schützen? Letztautorin Claudia Traidl-Hoffmann, Professorin für Umweltmedizin, rät, in den nächsten Monaten die Pollenflugvorhersagen zu Rate zu ziehen. Sie sagt: «Staubfiltermasken zu tragen, wenn die Pollenkonzentration hoch ist, kann das Virus und den Pollen gleichermaßen von den Atemwegen fernhalten.» 
Deutsche Dermatologische Gesellschaft e.V. (DDG)

\section{Fortgeschrittener schwarzer Hautkrebs: Immun- und zielgerichtete Therapie verhindern Rezidive und verlängern das Leben}

In Deutschland erkranken jedes Jahr etwa 23000 Menschen neu an schwarzem Hautkrebs. Anders als der weitaus häufigere und nicht lebensbedrohliche helle Hautkrebs ist das maligne Melanom für fast 3000 Todesfälle jährlich verantwortlich, denn es bildet deutlich häufiger Metastasen. Die Erkrankungszahlen des schwarzen Hautkrebses steigen seit einigen Jahren an. Professor Dr. med. Peter Elsner, Direktor der Klinik für Hautkrankheiten am Universitätsklinikum Jena, vermutet, dass dies auch mit der 2008 eingeführten Hautkrebs-Früherkennung zu tun hat. «Hautärztinnen und Hautärzte entdecken Melanome immer häufiger in einem frühen Stadium, was die Behandlungsmöglichkeiten und Heilungschancen verbessert. Tatsache ist aber auch, dass häufig immer noch sorglos mit den Themen Sonnenschutz und Solariumsnutzung umgegan- gen wird.» Die Risikofaktoren UV-Strahlung und Solarium würden offenbar noch immer unterschätzt, so der Beauftragte für die Öffentlichkeitsarbeit der Deutschen Dermatologischen Gesellschaft (DDG).

Wenn schwarzer Hautkrebs früh erkannt wird, kann die operative Entfernung des Tumors bei vielen Patientinnen und Patienten zur Heilung führen. «Je größer der Tumor ist und je weiter er sich ausgebreitet hat, umso mehr sinken die Heilungschancen», erklärt Professor Dr. med. Carola Berking, Direktorin der Hautklinik am Universitätsklinikum Erlangen und stellvertretende Vorsitzende der Arbeitsgemeinschaft Dermatologische Onkologie (ADO). Bis vor wenigen Jahren gab es für die Behandlung von Patientinnen und Patienten mit metastasiertem malignen Melanom keine Arzneimittel, die das Gesamtüberleben signifikant messbar verlängerten.
Das hat sich komplett geändert durch zwei wichtige neue Therapieprinzipien: Entdeckt wurden sogenannten Treibermutationen im Tumor, die für das Überleben und Wachstum der Krebszellen relevant sind.

Die zweite Entwicklung sind Krebs-Immuntherapien mit sogenannten CheckpointBlockern, die auch untereinander kombiniert werden.

Die Überlebenschancen können mit der Immuntherapie mit Checkpoint-Inhibitoren deutlich verbessert werden. «Schon die ersten Studienergebnisse mit diesen Antikörpern waren erfolgreich. Nun zeigen uns 5-JahresLangzeitdaten, dass die PD1-Checkpoint-Inhibitoren Nivolumab und Pembrolizumab das Gesamtüberleben bei Patientinnen und Patienten mit fortgeschrittenem bzw. metastasiertem Melanom auch nachhaltig signifikant verlängern», sagt Berking.

\section{Deutsche Dermatologische Gesellschaft e.V. (DDG)}

\section{Neue S2k-Leitlinie zur Teledermatologie}

Telemedizin ist eine zeitgemäße Ergänzung für die Versorgung von Patientinnen und Patienten mit Hauterkrankungen, wenn sie fachärztlichen Qualitätsstandards entspricht. Um diese im Bereich der Teledermatologie zu definieren, hat die Deutsche Dermatologische Gesellschaft e.V. (DDG) zusammen mit dem Berufsverband der Deutschen Dermatologen (BVDD) als erste Fachgruppe eine S2k-Leitlinie zu diesem Thema veröffentlicht, in der auf der Basis derzeit verfügbarer Evidenz Anwendungsfelder der Teledermatologie benannt und bewertet werden.

Ausgehend von mehreren Hundert ausgewerteten Studien gibt die neue S2k-Leitlinie Teledermatologie Empfehlungen, bei welchen Erkrankungen wie beispielsweise Psoriasis, Neurodermitis, Hautkrebs und chronischen Wunden teledermatologische Verfahren die Diagnose- und Therapieergebnisse verbessern.

«Teledermatologie sollten wir dort einsetzen, wo sie der Versorgung der Patientinnen und Patienten hilft, also frühe Diagnosen und Behandlungen möglich macht. Wir sehen gro- ße Chancen in der Telemedizin, um Therapie, Therapiemanagement, Nachsorge und die Patientenschulungen zu verbessern» sagt Professor Dr. med. Peter Elsner, Beauftragter für Öffentlichkeitsarbeit der Deutschen Dermatologischen Gesellschaft (DDG) und einer der Leitlinienautoren. Es gibt in der Dermatologie eine jahrzehntelange Erfahrung mit teledermatologischen Verfahren, aber bislang lag nur ein Leitfaden vor, nicht jedoch eine Leitlinie, die systematisch alle zur Verfügung stehenden und aussagekräftigen Studien erfasste und auswertete.

Untersucht haben die Leitlinienautorinnen und -autoren fünf häufige dermatologische Erkrankungen: Psoriasis, Neurodermitis, Hautkrebs, chronische Wunden und weitere Hautkrankheiten. Beim Thema Wundversorgung bestätigt die Leitlinie der Teledermatologie viele positive Effekte. Das beginnt bereits mit der Primärdiagnostik durch teledermatologische Verfahren, die empfohlen werden können, über die Verlaufskontrolle bis hin zur Schulung beim Verbändeanlegen durch Pflegepersonal.
Anders sieht es bei der Psoriasis aus. Eine Erstdiagnostik soll nicht allein auf der Basis eines teledermatologischen Befundes erfolgen; der Therapieverlauf kann aber gut mit einer Store-and-Forward-Technologie (SaF) oder der Realtime-Technologie begutachtet werden. Auch bei der Neurodermitis führt kein Weg an der Präsenzuntersuchung für die Erstdiagnostik herum. Beim Hautkrebs soll die Primärdiagnostik sowohl beim hellen als auch beim schwarzen Hautkrebs nicht allein aufgrund von KI-Lösungen erfolgen. «Die Daten zeigen, dass bisher noch die Präsenzdiagnostik, ggf. unterstützt durch digitale Techniken, der alleinigen digitalen Befundung überlegen ist», erklärt Professor Dr. med. Matthias Augustin, Leitlinienkoordinator und Direktor des Instituts für Versorgungsforschung in der Dermatologie und bei Pflegeberufen.

«Sollte die Leitlinie Teledermatologie andere Fachgruppen ermuntern, ihre Erkenntnisse zu telemedizinischen Anwendungen ebenfalls in Leitlinien zu überführen, würde uns das freuen», ergänzt Elsner.
108

Kompass Dermatol 2021;9:107-110

DOI: $10.1159 / 000515789$ 


\section{Heuschnupfen: Per App zum Klimaforscher werden}

Forschen vor der eigenen Haustür: Interessierte Bürgerinnen und Bürger sind ab sofort eingeladen, sich über eine kostenlose App aktiv an der Klimaforschung zu beteiligen. Der Clou: Wer mitmacht bekommt auch etwas zurück, nämlich beispielsweise Handwerkszeug, um mit einer Pollenallergie besser zurechtzukommen. Die App ist Teil des Verbundprojekts BAYSICS. Zuständig für den Bereich Pollen ist Geographie-Professorin Dr. Susanne JochnerOette von der Katholischen Universität Eichstätt-Ingolstadt (KU).

Dr. Susanne Jochner-Oette, Professorin für Physische Geographie / Landschaftsökologie und nachhaltige Ökosystementwicklung an der KU, erklärt den Zusammenhang so: «Bei höheren Temperaturen werden die Pollenkörner häufig in größeren Mengen produziert und früher freigesetzt. Zudem enthalten sie meist mehr Allergene und wirken deshalb aggressiver.»

Susanne Jochner-Oette und ihre Doktorandin Johanna Jetschni wollen hier helfen. Im Mittelpunkt steht dabei die kostenlose Web-App «BAYSICS». Die Nutzer werden durch wenige Klicks zu «Citizen Scientists» und helfen zum einendabei,eine RisikokartefürHeuschnupfen-
Geplagte zu erstellen, und zum anderen, Daten für die Erforschung des Klimawandels und seiner Auswirkungen zu sammeln.

Mitmachen ist denkbar einfach: Es genügen das eigene Smartphone oder PC und ein Gang vor die Haustür. Wo und wann blühen in meiner Heimat Birke, Hasel oder Gräser? Durch die kollektive Erfassung allergener Pflanzenarten entsteht nach und nach eine Risikokarte. So kann ein Pollenallergiker bald einfach nachschlagen, welche Gegenden er besser meiden sollte. Außerdem kann der Nutzer ein Tagebuch über seine Symptome führen.

Die Eichstätter Pollenforschung ist Teil des Verbundprojekts BAYSICS des Bayerischen Netzwerks für Klimaforschung. In mehreren Teilprojekten wird dort untersucht, wie sich der Klimawandel auf Pflanzen und Tiere in Bayern auswirkt. «Wir möchten dafür sensibilisieren, dass der Klimawandel überall stattfindet und auch in unserem Leben in Bayern deutliche Auswirkungen zeigt», sagt Prof. Dr. Susanne Jochner-Oette.

Besonders in der Region rund um Eichstätt und Ingolstadt hoffen Jochner-Oette und ihre Doktorandin Johanna Jetschni auf viele Teilnehmerinnen und Teilnehmer. Die beiden For-

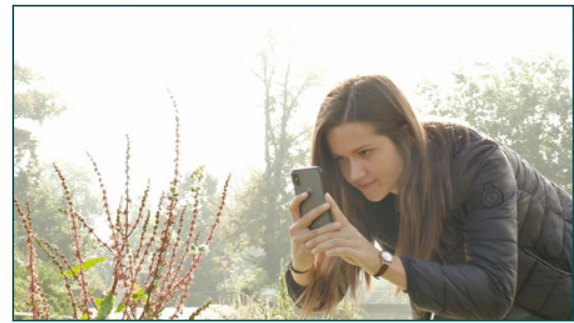

Über die BAYSICS-App können Bürgerinnen und Bürger nicht dabei helfen, Wissen über die Folgen des Klimawandels zu sammeln, sondern bekommen auch praktische Hilfe - etwa bei Pollenallergien. Foto: Nowak/upd

scherinnen haben hier ergänzend ein Pollenmessnetz installiert, um weitere Fragen zu bearbeiten. Unter anderem ist geplant, die Daten zur Pollenkonzentration mit den SymptomDaten aus der App zu vergleichen. So könne man abschätzen, ob eine hohe Belastung der Luft immer einhergeht mit einer hohen Belastung der Menschen. «Wenn zum Beispiel die Pollenkonzentration in der Luft gering ist, aber die Symptome beim Menschen stark, könnte das auf eine gestiegene Aggressivität der Pollen hindeuten», erklärt Jochner-Oette.

Wer mitmachen möchte, kann sich unter http://www.baysics.de die App herunterladen und seine Beobachtungen eintragen. Auch die Risikokarte für Allergiker findet sich dort.

\section{Medizinische Hochschule Hannover \\ Forschen für die Schwächsten}

Ebenso wie SARS-CoV-2 können auch andere Krankheitserreger für manche Menschen sehr gefährlich sein. Um Infektanfällige besser vor Viren und Bakterien schützen zu können, arbeiten mehr als 50 Forschungsteams unter Leitung der Medizinischen Hochschule Hannover (MHH) im Exzellenzclusters RESIST zusammen. Die VolkswagenStiftung und das Land Niedersachsen unterstützen RESIST nun mit einer Million Euro aus dem «Niedersächsischen Vorab». Das Geld nutzen 4 RESISTProfessorinnen und -Professoren für ihre Forschung. «Wir freuen uns sehr über die zusätzliche substanzielle Unterstützung der vier im Jahr 2020 neu berufenen RESIST-W2-Professorinnen und -Professoren, die unsere Forschungsbereiche mit ihrer Expertise bereichern, unterstützen und ergänzen», sagt RESIST-Sprecher Professor Dr. Thomas Schulz.
Professorin Dr. Sabrina Schreiner widmet sich den Adenoviren, die Bindehautentzündungen, Magen-Darm-Beschwerden oder Lungenentzündungen verursachen können. Diese Viren sind für Menschen mit einem geschwächten Immunsystem besonders gefährlich. Doch auch bei Gesunden können sie Lungenentzündungen auslösen, die tödlich verlaufen. Um neue Ansatzpunkte für die Entwicklung von Medikamenten und Impfstoffen zu finden, untersucht Professorin Schreiner im MHH-Institut für Virologie, wie sich das Virus in der Zelle vermehrt.

Um Lungenentzündungen dreht sich auch die Arbeit von Professor Dr. Nico Lachmann: Er erforscht bestimmte Immunzellen, die Bakterien und Viren beseitigen können - die sogenannten Fresszellen (Makrophagen). Sein Ziel ist es, dass Lungeninfektionen mit Hilfe von
Fresszellen geheilt werden können, die aus menschlichen Stammzellen im Labor gezüchtet werden. Professor Lachmann arbeitet in der MHH-Klinik für Pädiatrische Pneumologie, Allergologie und Neonatologie.

Von SARS-CoV-2 wissen wir inzwischen, dass Mutationen die Eigenschaften von Viren verändern können. Doch auch bei Bakterien ist dies der Fall. Welchen Einfluss Mutationen auf die krankmachende Wirkung von Bakterien und auf ihre Antibiotika-Resistenz haben das erforscht Professor Dr. Marco Galardini am Institut für Molekulare Bakteriologie des TWINCORE - Zentrums für Experimentelle und Klinische Infektionsforschung. Sein Ziel ist es, mit Hilfe der Bioinformatik und der Molekularbiologie vorhersagen zu können, wie sich die Unterschiede im Erbgut der Erreger weiterentwickeln. 


\section{Anerkennung beruflich bedingter Hauterkrankung zwingt nicht mehr zur Job-Aufgabe}

Hautkrankheiten, vor allem Handekzeme, führen mit Abstand die Statistik der gesetzlichen Unfallversicherung an. Eine Anerkennung als Berufskrankheit war bislang schwierig, es sei denn, man gab den Beruf auf. Das nun geänderte Berufskrankheitenrecht ermöglicht Betroffenen jetzt die Anerkennung als Berufskrankheit, auch wenn sie weiter in dem Beruf arbeiten.

Die Deutsche Dermatologischen Gesellschaft e.V. (DDG) weist darauf hin, dass das bewährte «Hautarztverfahren» mit seinem auf Behandlung und Prävention basierenden Ansatz nicht eingeschränkt werden sollte, damit keine «Präventionslücken» entstehen. Zum 1. Januar 2021 ist eine Änderung des Berufskrankheitenrechts in Kraft getreten. «Für die Beschäftigten steigt damit die Chance, dass ihre Erkrankung unabhängig davon, ob sie wegen der hautgefährdenden Tätigkeit den Job aufgeben oder ihre Tätigkeit fortsetzen, als Berufskrankheit anerkannt wird», erklärt Professor Dr. med. Peter Elsner, Direktor der Klinik für Hautkrankheiten am Universitätsklinikum Jena und Beauftragter für die Öffentlichkeitsarbeit der Deutschen Dermatologischen Gesellschaft (DDG).

«Die Abschaffung des Unterlassungszwangs wird zu einem großen Anstieg der Anerkennungszahlen führen», prognostiziert Professor Dr. med. Christoph Skudlik vom Institut für interdisziplinäre Dermatologische Prävention und Rehabilitation an der Universität Osnabrück. Der Anteil der Anerkennungen der fast 20000 gemeldeten BK 5101-Verdachtsfälle lag im Jahr 2019 mit nur 383 Fällen unter zwei Prozent. Bei den etwa 7500 BK-Meldungen zu Hautkrebsarten und ihren Vorstufen waren es mit 3766 Anerkennungen dagegen über 50 Prozent. «Wenn jetzt die Anerkennungsquote für die BK 5101 in die Tausende geht, wird sich das ganz erheblich auf die dermatologische Versorgung auswirken, da in all diesen Fällen dann die gesetzliche Unfallversicherung dauerhaft zuständig für die Behandlung ist», erklärt Skudlik, der auch Vorsitzender der Arbeitsgemeinschaft Berufsund Umweltdermatologie der DDG ist.

Mögliche Gefahren sieht der Experte darin, dass im Zuge dieser weitreichenden Reform das bewährte «Hautarztverfahren» eingeschränkt werden könnte.

«Wenn berufsbedingte Hauterkrankungen zu spät erkannt und behandelt werden, kann die Heilung sehr lange dauern. Patientinnen und
Patienten leiden stark unter den Belastungen, die mitunter dazu führen, dass der Beruf aufgegeben werden muss. Früh intervenieren ist das Handlungsgebot - das gut etablierte Hautarztverfahren sollte auch in Zukunft die Richtung weisen», betont Elsner. Mit der Gesetzesänderung bestehe die Gefahr einer Präventionslücke.

Die DDG-Experten begrüßen ausdrücklich, dass sich die gesetzliche Unfallversicherung erklärt hat, mit den Hautärztinnen und Hautärzten das gemeinsame Hautarztverfahren als effektives Präventionsverfahren auch bei Berufskrankheitenanzeigen fortzusetzen. Betroffene, die in hautbelastenden und gleichzeitig häufig auch systemrelevanten Berufen arbeiten, sollten dies nutzen und bei Hautbeschwerden möglichst frühzeitig zur Hautärztin oder zum Hautarzt gehen, um zu verhindern, dass ihre Erkrankung chronisch und ihre Berufsausübung gefährdet wird.

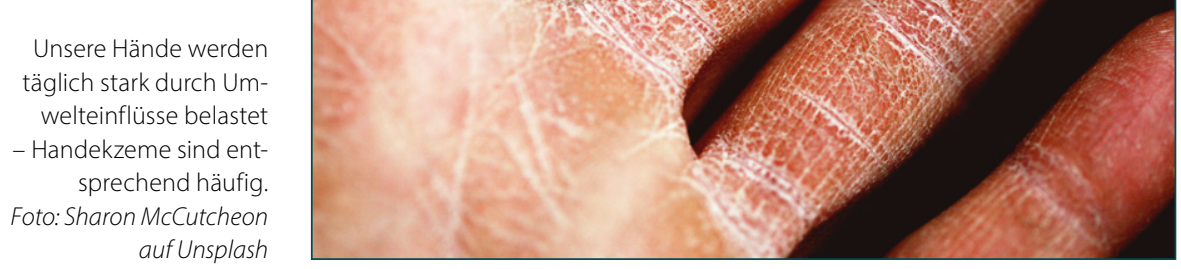

